\title{
Adenotonsillar hypertrophy and cor pulmonale: clinical and echocardiographic correlation
}

\author{
E.B. Kumar and N.S.V. Jaggarao \\ Department of Cardiology, King Fahad Hospital, P.O. Box 204, Al-Baha, Kingdom of Saudi Arabia
}

\begin{abstract}
Summary: It is well recognized that upper airways obstruction by adenotonsillar hypertrophy can lead to cor pulmonale, but delays in diagnosis still occur, leading to an appreciable morbidity and even occasional mortality. In the case presented, echocardiographic recognition of right ventricular enlargement and abnormal pulmonary valve motion served to complement and confirm the clinical diagnosis. Following surgical relief of the airways obstruction, the echocardiographic examination usefully documented the regression in right ventricular size and the return to normality of pulmonary valve motion.
\end{abstract}

\section{Introduction}

The clinical syndrome of chronic upper airways obstruction and cor pulmonale is well known, and includes noisy respiration, stridor, somnolence and adenotonsillar enlargement. Although the condition is often easily identified by simple examination of the upper airways, delays in diagnosis still occur, leading to cardiac failure and chronic respiratory infections. Signs of pulmonary hypertension are invariably present, but may be wrongly ascribed to underlying congenital cardiac anomalies. We report a case where the echocardiographic examination was valuable in excluding such anomalies, and usefully complemented the clinical assessment.

\section{Case report}

A 3 year old Saudi boy was admitted with a 7-day history of cough, dyspnoea and throat discomfort. He had experienced many similar previous episodes, suspected to be due to recurring bronchopneumonia. His mother remarked on his noisy breathing, particularly at night. He had also had several bouts of tonsillitis in the past, and had undergone adenoidectomy with bilateral myringotomy in February 1986. Tonsillectomy had not been performed.

Examination showed him to be ill-looking, febrile, coughing, tachypnoeic, with circumoral cyanosis, subcostal retraction and noisy breathing. The tonsils were considerably hypertrophied and reddened. The pulse was regular, with a rate of $135 /$ minute, and ausculta-

Correspondence: E.B. Kumar F.R.C.P. (Glasg)

Accepted:10 February 1989. tion revealed the presence of a third heart sound with gallop rhythm. Fine crepitations were heard throughout both lung fields. The liver was moderately enlarged, and tender to palpation. Cardiomegaly and pulmonary congestion were noted on the chest X-ray, while the electrocardiogram showed evidence of sinus rhythm, right axis deviation and right ventricular hypertrophy. There was a nutritional iron deficiency anaemia (haemoglobin $9.3 \mathrm{~g} / \mathrm{dl}$ ). Arterial $\mathrm{PO}_{2}$ on room air was $4.3 \mathrm{kPa}$ and $\mathrm{PCO}_{2} 4.9 \mathrm{kPa}$.

The child was initially given intravenous antibiotics, and later, on the basis of chest radiology, intravenous frusemide. He responded well to these measures and was much improved by the time of hospital discharge. The heart failure was thought to be secondary to recurrent respiratory tract infections; immunoglobulin deficiency was entertained, but this was not borne out by subsequent investigations.

Cross-sectional and M-mode echocardiography was then undertaken. The right ventricle was noted to be dilated (right ventricular diastolic dimension $2 \mathrm{~cm}$ ), and the pulmonary valve diastolic closure line was flat, with no ' $a$ ' descent, the appearances being suggestive of pulmonary hypertension (Figure 1). Left ventricular size and function were normal.

On the basis of these findings, and the clinical picture, it was thought likely that the child had developed cor pulmonale and pulmonary hypertension secondary to chronic upper airways obstruction from enlarged tonsils. He underwent uneventful tonsillectomy in June 1986. The echocardiogram was repeated two months after the operation, and showed a striking reduction in size of the right ventricle (right ventricular diastolic dimension $1.09 \mathrm{~cm}$ ). The pul-

(C) The Fellowship of Postgraduate Medicine, 1989 

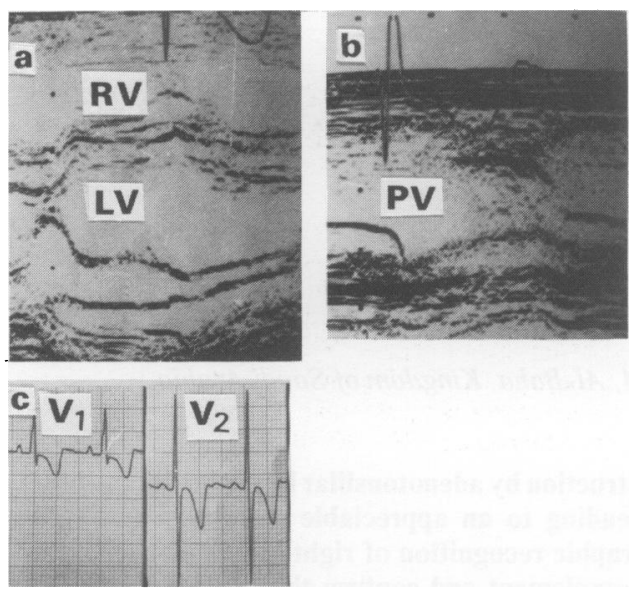

Figure 1 Pre-operative study: M-mode echocardiograms showing right ventricular dilatation (a), and abnormal pulmonary valve motion (b). RV, right ventricle; $L V$, left ventricle; PV, pulmonary valve. (c) Electrocardiogram showing right ventricular hypertrophy.

monary valve echogram was now noted to be normal (Figure 2). The electrocardiogram has also shown regression in the signs of right ventricular enlargement (Figure 2).

Since the operation, the child has remained well and active, with no signs of airways obstruction, and no clinical evidence of cor pulmonale.

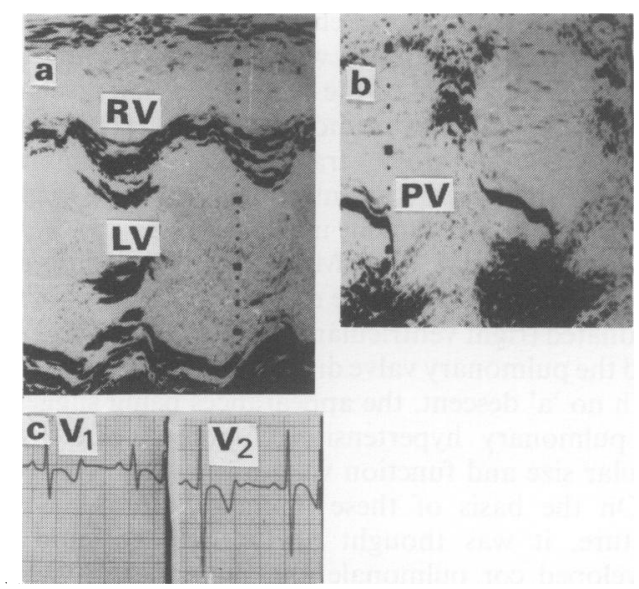

Figure 2 Post-operative study: M-mode echocardiograms showing reduction in right ventricular dimension (a), and normal pulmonary valve motion (b). RV, right ventricle; $L V$, left ventricle; $P V$, pulmonary valve. (c) Electrocardiogram showing regression in signs of right ventricular hypertrophy.

\section{Discussion}

The clinical features of the syndrome of cor pulmonale secondary to chronic upper airways obstruction have been well described in earlier reports, ${ }^{1,2}$ and includenoisy respiration, stridor, somnolence, adenotonsilla enlargement and signs of congestive cardiac failure? Mental retardation has also been noted in some series. ${ }^{3}$ There has been no previous echocardiographi study to our knowledge. Electrocardiographie evidence of right ventricular hypertrophy is nearl9 always found as well as clinical signs of pulmonary hypertension. Rarely, the child may be relativelg asymptomatic, and presents with cardiomegaly and pulmonary congestion. Most of the reported casesu were found in the age range of 2 to 4 years. $^{3}$

While signs of right ventricular enlargement with pulmonary hypertension, and clinical evidence of right heart failure appear to be an inevitable part of the syndrome, it is clear that left ventricular dysfunction can also occur, perhaps as a consequence of the combination of hypoxia and acidosis. ${ }^{4}$ Radiologicaf and clinical pulmonary oedema has been seen in most of the reported series; ${ }^{4,5}$ while its exact cause has not been established, of importance may well be the large swings in intrapleural pressure created by inspirator efforts against an obstructed upper airway, ${ }^{5,6}$ as well as. the sudden and acute rises in pulmonary artertbo pressure which can occur.

Greater awareness of this syndrome is cleatip necessary, since delays in diagnosis still occur. ${ }^{3}$ Dess pite several limitations, ${ }^{7,8}$ cross-sectional and M-mode echocardiography can be valuable in detecting righڤ̆ ventricular dilatation and abnormalities of pulmonars $\$$ valve motion suggestive of pulmonary hypertension $\overrightarrow{0}$ thus complementing the clinical examination.

It would seem that the echocardiographic recognition of these abnormalities may usefully serve tọ? confirm the clinical suspicion; furthermore, the technique would seem particularly valuable in excluding an. underlying congenital structural intracardiac anomalyo which might well have been suspected in the presence of the clinical signs described. Following relief of the respiratory obstruction, serial studies can clearly document the return to normality of right ventriculae dimension and pulmonary valve motion. The use of systolic time intervals for this purpose is more difficule and requires a complete recording of the pulmonary valve, ${ }^{7}$ while the chest $\mathrm{X}$-ray is known to be relatively insensitive in defining specific chamber enlargement $N$

Early detection and treatment can result in reversat of virtually all of the features of the condition. ${ }^{6}$ This syndrome should be considered in any child presenting with unexplained cardiac failure and recurring res piratory tract infection, particularly in the setting of adenotonsillar hypertrophy and stridor. ${ }^{9}$ 


\section{References}

1. Noonan, J.A. Reversible cor pulmonale due to hypertrophied tonsils and adenoids. (Abstract). Circulation 1965, 32 (suppl II): 164.

2. Menashe, V.D., Farrehi, C. \& Miller, M. Hypoventilation and cor pulmonale due to chronic upper airways obstruction. J Pediatr 1965, 67: 198-203.

3. Noonan, J.A. Pulmonary heart disease. Pediatr Clin North Am 1971, 18: 1262-1265.

4. Levy, A.M., Tabakin, B.S., Hanson, J.S. \& Narkewicz, R.M. Hypertrophied adenoids causing pulmonary hypertension and severe cardiac failure. $N$ Engl $J$ Med 1967, 277: 506-511.

5. Ainger, L.E. Large tonsils and adenoids in small children with cor pulmonale. Br Heart J 1968, 30: 356-362.
6. Cayler, G.G., Johnson, E.E., Lewis, B.E., Kortzborn, J.D., Jordan, J. \& Fricker, G.A. Heart failure due to enlarged tonsils and adenoids. Am J Dis Child 1969, 118: $708-717$.

7. Feigenbaum, H. In: Echocardiography, 3rd edition. Lea and Febiger, Philadelphia, 1981, pp. 211-216.

8. Lesser, L.M., Johnson, M., Felner, J.M. \& Hardison, J. Echocardiography in patients with suspected cor pulmonale. South Med J 1982, 75: 388-391.

9. Vellayappan, K., Ngiam, T.E. \& Low, P.S. Tonsillar hypertrophy, cor pulmonale and cardiac failure. Ann Acad Med Singapore 1981, 10: 461-465. 